\title{
ON SETS OF COMPLETENESS FOR FAMILIES OF HAAR FUNCTIONS
}

\author{
BY \\ J. J. PRICE AND ROBERT E. ZINK
}

1. Introduction. If one removes from a complete orthogonal family in $L^{2}(0,1)$ a nonempty set of functions, then the remaining set is, of course, no longer complete. Nevertheless, Talalyan [2] has shown that if any finite collection of functions is discarded from a complete set, then to every positive number $\varepsilon$ there corresponds a measurable set of measure $1-\varepsilon$ on which the remaining set of functions is complete. Moreover, in giving a simple proof of Talalyan's result, Goffman and Waterman [1] have observed that the same situation obtains if certain infinite collections are discarded. On the other hand, one may discard so many functions that the remaining family is complete on no set of positive measure. This is the case, for example, if one deletes from the Walsh system all functions save the Rademacher functions.

Thus, it is of interest to investigate the relation between the set of functions discarded and the sets of completeness for the remaining family. We begin here such an investigation. For the familiar system of Haar we are able to determine precisely those sets on which a given subsystem is complete. There follow some interesting corollaries. We note that the Rademacher functions comprise a lacunary subset of the Walsh system. Our results show that no lacunary subset of the Haar family can be complete on any set of positive measure. In the other direction, however, we are led to the following unexpected fact. The Haar system can be decomposed into an infinite number of subsystems, each of density zero with respect to the entire family, and each complete on a fixed set of measure $1-\varepsilon$.

Although they do not comprise an orthogonal set, the Schauder functions are also complete in $L^{2}(0,1)$. However, for the treatment of the problem herein considered, it is only the condition of completeness that is significant. In the last section of the article, we show that the technique earlier developed in order to study the Haar system can be applied to determine the sets of completeness for families of Schauder functions.

2. Preliminary considerations. It best suits our purposes to modify the customary definition of the Haar functions so that their supports are open sets.

Presented to the Society, July 20, 1964; received by the editors August 6, 1964. 
Thus, we let

$$
h_{0}=\chi_{(0,1)},
$$

and, for $m=k+2^{n}, n=0,1, \cdots, k=0, \cdots, 2^{n}-1$, we define

$$
h_{m}=2^{n / 2}\left[\chi_{r_{m}^{\prime}}-\chi_{I^{\prime \prime}{ }_{m}}\right] \text {, }
$$

where $I_{m}^{\prime}=\left(k \cdot 2^{-n},(2 k+1) 2^{-n-1}\right)$ and $I_{m}^{\prime \prime}=\left((2 k+1) 2^{-n-1},(k+1) 2^{-n}\right)$. Then, for each positive integer $m$, the support of $h_{m}$ is the union of two contiguous, congruent, open intervals. The notation has been chosen so that $h_{m}$ is positive on $I_{m}^{\prime}$ and negative on $I_{m}^{\prime \prime}$. The definition of the Haar functions groups them in blocks in a natural manner. For each nonnegative integer $n$, let

and let

$$
\mathscr{H}_{n+1}=\left\{h_{k}: 2^{n} \leqq k<2^{n+1}\right\},
$$

Then

$$
\mathscr{H}_{0}=\left\{h_{0}\right\} .
$$

$$
\mathscr{H}=\bigcup_{n=0}^{\infty} \mathscr{H}_{n}=\left\{h_{m}: m \geqq 0\right\}
$$

is the entire Haar system.

We also find it convenient to use a particularly simple Schauder basis for $C[0,1]$ differing slightly from the one originally given by Schauder. The basis in question is

where, for each $x$ in $[0,1]$,

$$
s_{-1}, s_{0}, s_{1}, \cdots
$$

$$
\begin{aligned}
s_{0}(x) & =\int_{0}^{x} h_{0}(t) d t, \\
s_{k+2^{n}}(x) & =2^{(n+2) / 2} \int_{0}^{x} h_{k+2^{n}}(t) d t,
\end{aligned}
$$

for $n \geqq 0$ and $0 \leqq k<2^{n}$, and

$$
s_{-1}=1-s_{0} .
$$

Our work depends on two basic theorems of Talalyan which are stated below without proof.

Definition 1. Let $G$ be a measurable subset of $[0,1]$. A system $\Phi$ of measurable functions defined and a.e. real valued on $G$ is said to be total in measure if for each measurable $f$ defined on $G$ there is a sequence of finite linear combinations of elements of $\Phi$ that converges in measure to $f$.

Definition 2. Let $G$ be a measurable subset of $[0,1]$. A system $\Phi$ of elements of $L^{2}(G)$ is total in $L^{2}$ if for each $f$ in $L^{2}(G)$ there is a sequence of finite linear combinations of elements of $\Phi$ that converges in mean to $f$. 
Such a system is also said to be closed in $L^{2}$. We recall that the concepts of completeness and closure (totality) coincide in $L^{2}$.

THEOREM 3. Let $G$ be a measurable subset of $[0,1]$. In order that a system $\Phi$ be total in measure on $G$ it is both necessary and sufficient that to each positive number $\varepsilon$ there should correspond a measurable set $E$ contained in $G$ such that $\mu(E) \geqq \mu(G)-\varepsilon$ and $\Phi$ is total in $L^{2}(E)$.

THEOREM 4. Let $G$ be a measurable subset of $[0,1]$. If $\left\{\phi_{n}\right\}$ is a sequence of measurable functions defined and a.e. real valued on $G$, and if $\left\{\phi_{n}\right\}$ is total in measure, then it remains so when a finite set of functions is removed from it.

3. Sets of completeness for families of Haar functions. The following theorem is the key to the entire discussion. In conjunction with the results of Talalyan it yields a complete description of the situation.

THEOREM 5. Let $\Phi=\left\{\phi_{n}\right\}$ be a set of Haar functions. For each $n$, let $E_{n}$ be the support of $\phi_{n}$, and let $E=\limsup _{n} E_{n}$. Then $\Phi$ is total in measure on a measurable set $G$ if and only if $\mu(G)=\mu(G \cap E)$.

Proof of necessity. Suppose that $\left\{\phi_{n}\right\}$ is total in measure on $G$. We shall show that $S=G \cap(I-E)$ is a null set.

For each $x$ in $S$, there is an integer $k(x)$ such that

$$
x \notin \bigcup\left\{E_{n}: n>k(x)\right\} .
$$

Let

$$
S_{n}=\{x: x \in S \wedge k(x)=n\}
$$

According to Theorem 4 , if $N$ is any natural number, then $\left\{\phi_{n}: n>N\right\}$ is also total in measure on $G$. Thus, there exists a sequence $\left\{f_{k}\right\}$ of finite linear combinations of these functions that converges in measure to $g$, the characteristic function of $S_{N}$. Now $S_{N} \cap E_{N+n}=\varnothing$ for every natural number $n$; hence, for every $k, S_{N}$ has no point in common with the support of $f_{k}$. Therefore,

$$
\mu\left(\left\{x:\left|g(x)-f_{k}(x)\right| \geqq 1\right\}\right) \geqq \mu\left(S_{N}\right) .
$$

From the definition of convergence in measure, it thus follows that each $S_{N}$ is a null set. Finally, $S=\bigcup_{n=1}^{\infty} S_{n}$ so that $S$ is also a set of measure zero.

Proof of sufficiency. We assume that $E$ has positive measure, since in the contrary case there is nothing to prove. It will suffice to prove that $\Phi$ is total in measure on $E$, for this implies that $\Phi$ has the same property relative to any measurable subset of $E$. To do this we need show only that the characteristic function of each measurable subset of $E$ can be arbitrarily closely approximated in measure by finite linear combinations of elements of $\Phi$, for the set of all such characteristic functions is total in measure on $E$. 
Let $A$ be a nonnull measurable subset of $E$, let $g$ be the characteristic function of $A$ and let $\varepsilon$ be any positive number. We first choose an open set $U$ containing $A$ and satisfying the inequality $\mu(U-A)<\varepsilon / 2$. We then proceed to construct a finite linear combination $f$ of elements of $\Phi$ such that the support of $f$ is a subset of $U$ and

$$
\mu(\{x: f(x)=1\}) \geqq \mu(A)-\varepsilon / 2 .
$$

Such a function will differ from $g$ on a set of measure less than $\varepsilon$.

Let $\left\{\varepsilon_{n}\right\}$ be a sequence of positive numbers so chosen that

$$
\sum_{n=1}^{\infty} \varepsilon_{n}<\varepsilon / 4 \mu(A)
$$

Since $A$ is a subset of limsup $E_{n}$, each point of $A$ belongs to infinitely many members of $\left\{E_{n}: n \geqq 1\right\}$, and it follows that $\left\{E_{n}: E_{n} \subset U\right\}$ is a covering of $A$ in the sense of Vitali. Hence, by the Vitali covering theorem, there exist disjoint sets $E_{n_{1}}, \cdots, E_{n_{k_{1}}}$ such that

$$
\mu\left(A-\bigcup_{j=1}^{k_{1}} E_{n_{j}}\right)<\varepsilon_{1} \mu(A) .
$$

As a first approximation to $g$, take

$$
f_{1}=\sum_{j=1}^{k_{1}} a_{j} \phi_{n_{j}}
$$

where $a_{j}$ is $+\left(\max \phi_{n_{j}}\right)^{-1}$ or $-\left(\max \phi_{n_{j}}\right)^{-1}$ according as $\mu\left(A \cap E_{n_{j}}^{\prime}\right)$ does or does not exceed $\mu\left(A \cap E_{n_{j}}^{\prime \prime}\right)$. Then, $f_{1}$ is a step function vanishing on the complement of $U$ and assuming the values +1 and -1 on the open sets $S_{11}$ and $S_{12}$. If

$$
A_{1}=A \cap \bigcup_{j=1}^{k_{1}} E_{n_{j}}
$$

then

$$
\mu\left(S_{11} \cap A_{1}\right)=\alpha \mu\left(A_{1}\right)>(1 / 2) \mu\left(A_{1}\right)>(1 / 2)\left(1-\varepsilon_{1}\right) \mu(A) .
$$

We now improve our approximation to $g$ by constructing a function $f_{2}$ vanishing on $S_{11}$ and such that $f_{1}+f_{2}$ assumes the value +1 on a set that contains a subset of $A$ having measure approximately equal to $(3 / 4) \mu(A)$.

Again taking account of the fact that we are dealing with a subset of $\limsup _{n} E_{n}$, we observe that $\left\{E_{n}: E_{n} \subset S_{12} \wedge n>n_{k_{1}}\right\}$ is a Vitali covering of $A \cap S_{12}$. Thus, another application of the Vitali covering theorem yields a finite sequence of disjoint sets $E_{n_{k_{1}+1}}, \cdots, E_{n_{k_{2}}}$ for which

$$
\mu\left(A \cap S_{12}-\bigcup_{j=k_{1}+1}^{k_{2}} E_{n_{j}}\right)<\varepsilon_{2} \mu\left(A \cap S_{12}\right) .
$$


Following the pattern established above, we define

$$
f_{2}=\sum_{j=k_{1}+1}^{k_{2}} a_{j} \phi_{n_{j}},
$$

where, for $k_{1}<j \leqq k_{2}, a_{j}=+2\left(\max \phi_{n_{j}}\right)^{-1}$, if $\mu\left(A \cap E_{n_{j}}^{\prime}\right)$ exceeds $\mu\left(A \cap E_{n_{j}}^{\prime \prime}\right)$, and $a_{j}=-2\left(\max \phi_{n_{j}}\right)^{-1}$ in the contrary case. Now $f_{2}$ is a step function assuming the values +2 and -2 on the open sets $S_{21}$ and $S_{22}$, and, since $S_{21}$ and $S_{22}$ are subsets of $S_{12}, f_{1}+f_{2}$ takes the value +1 on $S_{11} \cup S_{21}$. Moreover,

$$
\begin{aligned}
\mu\left(S_{11} \cup S_{21}\right) & \geqq \mu\left(A \cap\left(S_{11} \cup S_{21}\right)\right) \\
& =\mu\left(A \cap S_{11}\right)+\mu\left(A \cap S_{21}\right) \\
& =\alpha \mu\left(A_{1}\right)+\mu\left(A \cap S_{21}\right) \\
& >\alpha\left(1-\varepsilon_{1}\right) \mu(A)+(1 / 2)\left(1-\varepsilon_{2}\right)(1-\alpha) \mu\left(A_{1}\right) \\
& >\left[(3 / 4)-\left(\varepsilon_{1}+\varepsilon_{2}\right)\right] \mu(A) .
\end{aligned}
$$

Proceeding inductively, we thus construct a sequence $\left\{f_{n}\right\}$ of finite linear combinations of elements of $\Phi$ such that the support of each $f_{n}$ is contained in $U$ and

$$
\begin{aligned}
\mu\left(\left\{x: \sum_{k=1}^{n} f_{k}(x)=1\right\}\right) & \geqq \mu\left(\left\{x: \sum_{k=1}^{n} f_{k}(x)=1\right\} \cap A\right) \\
& >\left[\left(1-2^{-n}\right)-\sum_{i=1}^{n} \varepsilon_{i}\right] \mu(A) .
\end{aligned}
$$

Hence, if $N$ is so large that $2^{-N} \mu(A)<\varepsilon / 4$ and if $f=\sum_{i=1}^{N} f_{i}$, then

$$
\mu(\{x: f(x)=1\})>\mu(A)-\varepsilon / 2,
$$

and the desired result has been obtained.

From Theorem 5 several other results can be easily deduced. The first of these is an immediate consequence of this theorem and Theorem 3.

Corollary 6. A set $\Phi$ of Haar functions is complete on some set of positive measure if and only if limsup $E_{n}$ has positive measure.

Indeed, a stronger, though less elegant, assertion can be made.

COROllary 7. Let $\Phi=\left\{\phi_{n}\right\}$ be a set of Haar functions, and, for each $n$, let $E_{n}$ be the support of $\phi_{n}$. In order that $\Phi$ be complete on a set of measure $a-\varepsilon$ for every positive $\varepsilon$, it is both necessary and sufficient that limsup ${ }_{n} E_{n}$ have measure a or larger.

COROLlaRY 8. In order that a set $\left\{\phi_{n}\right\}$ of Haar functions be complete on some set of positive measure it is necessary that the series $\sum_{n=1}^{\infty} \mu\left(E_{n}\right)$ diverge. 
Proof. If $\left\{\phi_{n}\right\}$ is complete on some set of positive measure, then, by virtue of Corollary $6, \limsup _{n} E_{n}$ has positive measure. Now

so that

$$
\limsup E_{n}=\bigcap_{n=1}^{\infty} \bigcup_{k=n}^{\infty} E_{k}
$$

$$
\begin{aligned}
0<\mu\left(\limsup _{n} E_{n}\right) & \leqq \mu\left(\bigcup_{k=n}^{\infty} E_{k}\right) \\
& \leqq \sum_{k=n}^{\infty} \mu\left(E_{k}\right),
\end{aligned}
$$

for each natural number $n$, from which the divergence of the given series follows.

We remark that the converse of Corollary 8 is false. The following example is included in the interests of completeness.

EXAMPLE 9. For each natural number $n$, let

$$
\Phi_{n}=\bigcup_{k=2^{n}}^{2^{n+1-1}}\left\{f: f \in \mathscr{H}_{k} \wedge \text { support }(f) \subset\left(0,2^{-n}\right)\right\},
$$

and let $\Phi=\bigcup_{n=1}^{\infty} \Phi_{n}$. If $E_{n}$ is the support of the $n$th element of $\Phi$ (according to some scheme of enumeration), then

$$
\sum_{n=1}^{\infty} \mu\left(E_{n}\right)=\sum_{n=1}^{\infty} \Sigma\left\{\mu\left(E_{k}\right): \phi_{k} \in \Phi_{n}\right\}=\sum_{n=1}^{\infty} a_{n}
$$

diverges, since each $a_{n}$ is 1 . Because limsup $E_{n}$ is empty, $\Phi$. is complete on no set of positive measure.

CoRollary 10. A lacunary subset of the Haar system is complete on no set of positive measure.

Proof. Let $\Phi=\left\{\phi_{k}\right\}$ be a set of Haar functions, let $Z^{+}$be the set of nonnegative integers and let $f: Z^{+} \rightarrow Z^{+}$be the function satisfying $f(n)=\operatorname{card}\left(\Phi \cap \mathscr{H}_{n}\right)$, for all $n$ in $Z^{+}$. By virtue of Corollary 8 , if $f$ is bounded, then $\Phi$ cannot be complete on any set of positive measure. For

$$
\sum_{k=1}^{\infty} \mu\left(E_{k}\right) \leqq B\left[1+\sum_{n=0}^{\infty} 2^{-n}\right]
$$

where $B$ is an upper bound for $f$.

Now suppose that $\Phi=\left\{h_{n_{i}}\right\}$, where

$$
n_{i+1} / n_{i} \geqq \lambda>1 .
$$

Since it is easy to see that $f(n) \leqq[K]$, for all $n$, where $\lambda^{R}=2$, the corollary is proved.

We conclude this section with an interesting decomposition of the Haar system 
into infinitely many sparsely distributed subsystems each of which is complete on a fixed set of large measure.

COROLlaRY 11. There exists an infinite partition $\left\{\Phi_{i}\right\}$ of the Haar system such that to each positive $\varepsilon$ there corresponds a measurable set of measure greater than $1-\varepsilon$ on which each $\Phi_{i}$ is complete and such that each of the sets $\left\{n: h_{n} \in \Phi_{i}\right\}$ has zero density.

Proof. Partition the natural numbers into an infinite number of infinite subsets $S_{i}=\left\{n_{i k}: k=1,2, \cdots\right\}, i=1, .2, \cdots$, in such a way that

$$
n_{i k} \geqq 2 n_{i, k-1},
$$

for all $i$ and all $k$ greater than 1 . In order to form the set $\Phi_{i}$ we select functions from the blocks $\mathscr{H}_{n_{i 1}}, \mathscr{H}_{n_{i 2}}, \cdots$ according to the following scheme. Let

$$
\mathscr{A}_{i, k+2^{m}}=\left\{f: f \in \mathscr{H}_{n_{i, k+2^{m}}} \wedge \operatorname{support}(f) \subset\left(k \cdot 2^{-m},(k+1) 2^{-m}\right)\right\},
$$

for $m=0,1, \cdots$ and $k=0, \cdots, 2^{m}-1$, and let

$$
\Phi_{i}=\bigcup_{j=1}^{\infty} \mathscr{A}_{i j} \text {. }
$$

It is easy to see that the superior limit of the sequence of supports of functions in $\Phi_{i}$ contains all points of $[0,1]$ save the dyadic rationals; hence, each $\Phi_{i}$ is complete on measurable sets having measure arbitrarily close to 1 .

We show next that $\Phi_{i}$ has zero density in the Haar system. For each natural number $k$, let

$$
N_{i}(k)=\operatorname{card}\left(\left\{n: h_{n} \in \Phi_{i} \wedge n \leqq k\right\}\right) .
$$

Clearly, the function thus defined is constant on each block $Z_{n}$ of integers that corresponds to a block $\mathscr{H}_{n}$, where $n$ is not one of the $n_{i j}$. If $2^{n} \leqq r<2^{n+1}$, then in the block of integers $Z_{n_{i r}}$ there is a chain of length $2^{-n+n_{i r}}$ on which the function increases steadily. At the last element of this chain, $k_{i r}$, the density function has a local maximum. Hence, in order to show that

it will suffice to show that

$$
\lim _{k} N_{i}(k) / k=0,
$$

$$
\lim _{j}\left[N_{i}\left(k_{i j}\right) / k_{i j}\right]=0 .
$$

To this end, note first that $N_{i}\left(k_{i j}\right)$ is certainly less than the sum of the number of elements in $\mathscr{A}_{i j}$ and the number of elements in $\bigcup_{k=1}^{n_{i}, j-1} \mathscr{H}_{k}$. Thus, if $2^{n} \leqq j<2^{n+1}$, then

$$
\begin{aligned}
N_{i}\left(k_{i j}\right) & \leqq 2^{-n+n_{i j}}+\sum_{k=1}^{n_{i}, j-1} 2^{k} \\
& <2^{-n+n_{i j}}+2\left(2^{n_{i, j-1}}\right)
\end{aligned}
$$


Next observe that

$$
k_{i j} \geqq 2^{-1+n_{i j}},
$$

because $k_{i j}$ is an element of $Z_{n_{i}}$.

Hence,

$$
\begin{aligned}
N_{i}\left(k_{i j}\right) / k_{i j} & <2^{1-n_{i j}}\left(2^{-n+n_{i j}}+2^{1+n_{i, j-1}}\right) \\
& <2^{1-n}+2^{2-n_{i, j-1}} \\
& <2^{1-n}+2^{2-2 j^{j-1}} \\
& <6 / 2^{n}
\end{aligned}
$$

since $n_{i j} \geqq 2 n_{i, j^{-1}}$ and $n_{i j} \geqq 2^{j}$, and the density assertion follows.

Finally, we enumerate the functions in $\mathscr{H}-\bigcup_{i=1}^{\infty} \Phi_{i}$ and adjoin the $k$ th element of this set to $\Phi_{k}$. Since the augmented subfamilies have the required properties, they comprise a partition of $\mathscr{H}$ of the desired type.

4. Sets of completeness for families of Schauder functions. Since the family $\left\{s_{n}\right\}$ of Schauder functions is closely related to the Haar system, it is reasonable to suppose that theorems similar to those established in section 3 hold also for this system. To see that this is indeed the case, one need only establish the analogue of Theorem 5 and then observe that each of the corollaries of Theorem 5 has a corresponding parallel in the Schauder system.

THEOREM 12. Let $\Phi=\left\{\phi_{n}\right\}$ be a subset of the Schauder system, let $E_{n}$ be the support of $\phi_{n}$ and let $E=\limsup _{n} E_{n}$. Then, $\Phi$ is a total in measure on $E$.

Although the technical details are somewhat different, the argument required to prove Theorem 12 follows the pattern established in the demonstration of Theorem 5.

Finally, we remark that the same arguments used to establish the various corollaries of Theorem 5 can be employed to prove for the Schauder system exact analogues of those propositions.

\section{REFERENCES}

1. C. Goffman and D. Waterman, Basic sequences in the space of measurable functions, Proc. Amer. Math. Soc. 11 (1960), 211-213.

2. A. A. Talalyan, On the convergence almost everywhere of subsequences of partial sums of general orthogonal series, Izv. Akad. Nauk Armyan SSR Ser. Fiz.-Mat. Nauk 10 (1957), 17-34. (Russian. Armenian summary).

PURDUB UNIVERSITY,

LAFAYETTE, INDIANA

INSTITUTB FOR ADVANCED STUDY, Princeton, NEW JeRseY 